\title{
Relación entre estilos de aprendizaje en ambientes a distancia e inteligencia emocional en alumnos de licenciatura
}

\author{
Relação entre estilos de aprendizagem em \\ ambientes remotos e inteligência emocional em \\ estudantes de bacharelado \\ Relationship between learning styles in remote \\ environments and emotional intelligence in \\ undergraduate students
}

\author{
ARMANDO LOZANO-RODRÍGUEZ (iD) \\ FERNANDA INÉZ GARCÍA-VÁZQUEZ ${ }^{b}$ \\ JOSÉ LUIS GARCÍA-CUÉc
}

Resumen

La manera en la que un discente aprende depende de diversos factores como los familiares, ambientales, personales, cognitivos y emocionales. Un estudiante

a Instituto Tecnológico de Sonora (ITSON), Ciudad Obregón, Sonora, México. Doctor en Innovación y Tecnología Educativa, e-mail: armando.lozano@itson.edu.mx

b Instituto Tecnológico de Sonora (ITSON), Ciudad Obregón, Sonora, México. Doctora en Ciencias Sociales, e-mail: fernanda.garcia@itson.edu.mx

' Colegio de Posgraduados (CP), Campus Montecillo, Texcoco, Estado de México, México. Doctor en Ciencias de la Educación, e-mail: jlgcue@colpos.mx 
puede preferir una forma de aprender, lo cual está relacionada directamente con su estilo de aprendizaje; pero también puede formarse mejor dependiendo de las emociones que presente a la hora del acto mismo de educarse. Si a lo anterior se les añade la incorporación de TIC en programas educativos en modalidades a distancia, el panorama adquiere otro matiz distinto. Por lo anterior, la presente investigación tuvo como propósito relacionar los Estilos de Aprendizaje en ambientes a distancia con algunos factores de Inteligencia Emocional de alumnos de pregrado de una Universidad del Sur de Sonora, México. La investigación fue de tipo cuantitativa, no experimental y correlacional. La muestra fue de 193 estudiantes universitarios de diversas carreras matriculados en agosto de 2018. Se utilizaron dos cuestionarios el Quirón Test para los Estilos de Aprendizaje y el Cuestionario de Inteligencia Emocional-CIE. Se analizaron los datos por estadísticos descriptivos, pruebas de normalidad, análisis de correlación y comparación de dos muestras independientes. Se identificó que los estudiantes que obtienen mayores valores en los Estilos de Aprendizaje Analítico, Global, Dependiente, Teórico y Práctico obtienen menores valores en las cuatro dimensiones emocionales. Los discentes con mayores puntuaciones en el Estilo de Aprendizaje Verbal tienen menores valores en Autorregulación, Autoeficacia y Empatía. También, el género y el tipo de carrera influyeron en los valores obtenidos. Se concluyó que hay relación entre Estilos de Aprendizaje e Inteligencia Emocional.

Palabras clave: Estilos de aprendizaje. Inteligencia emocional. Aprendizaje. Licenciatura

\section{Resumo}

A maneira pela qual um aluno aprende depende de vários fatores, como família, meio ambiente, pessoal, cognitivo e emocional. Um aluno pode preferir uma maneira de aprender, diretamente relacionada ao seu estilo de aprendizagem; mas também pode ser formado melhor, dependendo das emoções que apresenta no momento do ato de educar-se. Se a incorporação das TIC nos programas educacionais nas modalidades a distância é acrescentada ao exposto, o panorama assume uma nuance diferente. Portanto, o objetivo desta pesquisa foi relacionar os Estilos de Aprendizagem em ambientes remotos com alguns fatores de Inteligência Emocional de estudantes de graduação de uma Universidade do Sul de Sonora, Mexico. A pesquisa foi quantitativa, não experimental e correlacional. A amostra foi composta por 193 universitários de várias carreiras matriculadas em agosto de 2018. Foram utilizados dois questionários: o Teste Chiron para Estilos de Aprendizagem e o Questionário de Inteligência Emocional-CIE. Os dados foram analisados por estatística descritiva, testes de normalidade, análise de correlação e comparação de duas amostras independentes. Identificou-se que os alunos que obtêm valores mais altos nos Estilos de Aprendizagem Analítico, Global, Dependente, Teórico e Prático obtêm valores mais baixos nas quatro dimensões emocionais. Os alunos com notas mais altas no Estilo de Aprendizagem Verbal têm valores mais baixos em Autorregulação, Autoeficácia e Empatia. Além disso, 
sexo e tipo de carreira influenciaram os valores obtidos. Concluiu-se que existe uma relação entre Estilos de Aprendizagem e Inteligência Emocional.

Palavras-chave: Estilos de aprendizagem. Inteligência emocional. Aprendizagem. Bacharelado.

\begin{abstract}
The way in which a student learns depends on various factors such as family, environmental, personal, cognitive and emotional. A student may prefer a way of learning, which is directly related to his learning style; but it can also be formed better depending on the emotions it presents at the time of the act of educating itself. If the incorporation of ICT in educational programs in distance modalities is added to the above, the panorama takes on a different nuance. Therefore, this research was aimed to relate the learning styles in remote environments with some Emotional Intelligence factors of undergraduate students of Southern Sonora University in Mexico. The research was quantitative, non-experimental and correlational. The sample consisted of 193 university students from various careers enrolled in August 2018. Two questionnaires were used: The Chiron Test for Learning Styles and the Emotional Intelligence Questionnaire (EIQ). Data were analyzed by descriptive statistics, normality tests, correlation analysis and comparison of two independent samples. It was identified that students who earn higher values in Analytical Learning Styles, Global, dependent, theoretical and practical values obtained lower values in the four emotional dimensions. Students with higher scores in the Verbal Learning Style have lower values in Self-regulation, Self-efficacy and Empathy. Also, gender and type of career influenced the values obtained. It was concluded that there is a relationship between Learning Styles and Emotional Intelligence.
\end{abstract}

Keywords: Learning styles. Emotional Intelligence. Learning. Undergraduate.

\title{
Introducción
}

La manera en la que un estudiante aprende está afectada por un sinnúmero de diversos factores (CORNEJO CHÁVEZ \& REDONDO ROJO, 2007; Ramirez Dorantes, 2016). Los hay familiares, ambientales, personales, cognitivos e incluso emocionales (ZUO, WEAVER, MACRAE, \& WANG, 2019). Los familiares refieren a la situación en la que el aprendiz está viviendo en casa (MOESTUE \& HUT'TLY, 2008; IBABE, 2016). Los ambientales están relacionados con el clima en el salón de clase, las condiciones físicas que rodean el escenario educativo (BOYKIN; BAILEY, 2000). Los personales hacen alusión a la manera en la que el 
mismo aprendiz manifiesta su propia personalidad, sus relaciones con los otros, incluso hasta su propio estado físico de salud (Torres Valenzuela, Velázquez Hernández, Martínez Luna, García García, Gómez Rodríguez, Jasso Jiménez, \& Ortiz Valdivia, 2018). Los cognitivos están relacionados con las capacidades intelectuales del aprendiz, la manera en la que recibe y procesa información (BOTHAINA, HAMOUDA, \& ABDELLA, 2019). Los emocionales refieren a las distintas maneras en las que el aprendiz manifiesta sus propias emociones y puede reconocerlas en los demás (MAYRING, 2007; HANIN \& VAN NIEUWENHOVEN, 2016).

Un aprendiz puede preferir una forma de aprender, lo cual está relacionado directamente con su estilo de aprendizaje; pero también puede aprender más rápido o más lento dependiendo de las emociones que presente a la hora del acto mismo de aprendizaje (BETTIS-OUTLAND \& GUILLORY, 2018). Por lo anterior, el presente estudio busca posibles relaciones entre las preferencias que tienen los estudiantes para aprender y algunas de las habilidades emocionales que se desprenden del constructo de lo que se denomina inteligencia emocional. Si lo anterior se le añade el rubro de "a distancia", el panorama adquiere otro matiz distinto al presencial, debido al uso actual que se le ha dado a las tecnologías de información y comunicación (TIC).

Hay que reconocer que las TIC se han convertido en una herramienta necesaria como complemento educativo; sin embargo, estas tecnologías no pueden desligarse del uso de internet, aunado en gran medida por las redes sociales. A la fecha, las redes sociales se han generalizado más en los jóvenes y adolescentes, quienes pertenecen a una generación nativa de la tecnología (DE LA HOZ, ACEVEDO \& TORRES, 2015).

El aumento en el uso de las redes sociales se ha convertido en un fenómeno común en los últimos años generando gran impacto en el desarrollo de formas de interacción y socialización entre los estudiantes. Al respecto, The Statistics Portal reporta que en 2015 Facebook fue la red más utilizada en el mundo, con más de un billón de usuarios registrados, mientras que Twitter alcanzó más de 284 millones (PÉREZ-ALCALÁ, ORTIZ-ORTIZ \& FLORES-BRISEÑO, 2015). A pesar de que las redes sociales pueden ser una herramienta perfecta para el aprendizaje, son 
pocos los estudiantes que las usan para propósitos escolares (EMI-KAKUSHI \& MARTÍNEZ-ÉVORA, 2016), optando en la gran mayoría de los casos, por patrones de uso orientados hacia el entretenimiento.

La juventud es una continuación del período de separación e individualización en que se adquieren autonomía con respecto a la toma de decisiones; por lo tanto, se considera que este grupo de personas son más propensos a la interferencia de la Internet en su vida cotidiana, siendo un factor importante para la comprensión del uso de las redes sociales y la influencia que pueda generar en su comportamiento (ASSUNÇÃO \& MATOS, 2014).

$\mathrm{Al}$ hacer una revisión de fuentes documentales, para esta investigación, se destacaron dos diferentes áreas: Estilo de Aprendizaje e Inteligencia Emocional.

El concepto de estilo de aprendizaje está relacionado con la identificación de patrones de comportamiento que tienden a repetirse cada vez que se presentan ciertas condiciones en el salón de clase (GUILD \& GARGER, 1998). También, los estilos están asociados a las preferencias que se tienen sobre las diversas maneras en que se percibe, procesa, almacena y recupera información (GRIGORENKO \& STERNBERG, 1997). Estas preferencias permean en las prácticas educativa de muchas maneras. Dunn y Dunn (1998) parten del supuesto de que en la medida en que los estilos sean considerados en los procesos de enseñanza, mayor posibilidad habrá de que los estudiantes aprendan.

Las formas y disposiciones en las que a un alumno le gusta aprender son llamadas preferencias. Cuando esas preferencias son evidenciadas de manera repetida se pueden identificar patrones de comportamiento. Esos patrones pueden ser descritos y medidos a través de lo que se conoce como estilo de aprendizaje (ALONSO, GALLEGO \& HONEY, 1994). Existe al respecto una gran cantidad de teorías que abordan los estilos de aprendizaje atendiendo diferentes niveles educativos. Los hay para estudiantes de primaria (DUNN \& DUNN, 1998), para secundaria y preparatoria (FLEMING \& MILLS, 1992), para pregrado y posgrado (CASSIDY, 2004).

Curry (1983) propuso una clasificación de teorías de estilos cognitivos y de aprendizaje haciendo una analogía con las capas de una cebolla. La capa externa 
tiene que ver con las preferencias instruccionales, aquella en la que los estudiantes eligen el ambiente más idóneo para aprender. Esta primera capa es la más variable de todas. La siguiente capa tiene que ver con las preferencias para procesar información. El aprendiz no es afectado tanto por el ambiente y en consecuencia, el estilo es más estable. La tercera capa es la más profunda y tiene que ver con el estilo cognitivo y de personalidad. Difícilmente el estilo va a cambiar a lo largo de la vida.

Las preferencias instruccionales fueron tratadas por Grasha (2002). Su modelo tiene que ver con tres pares opuestos de estilos de aprendizaje (Tabla 1). El primer par tiene que ver con la acción que puede tomar (o no) el alumno para interactuar en el salón de clase. El segundo tiene que ver con la manera en que se relaciona con sus compañeros de clase; y el tercero con su nivel de autonomía en su desempeño académico.

Tabla 1 - Estilos de aprendizaje propuestos por Anthony Grasha (2002)

\begin{tabular}{|l|l|}
\hline $\begin{array}{l}\text { Estilos de } \\
\text { aprendizaje }\end{array}$ & Características de los aprendices que posee cada estilo \\
\hline 1. Participativo & Manifiestan interés en la clase. Son entusiastas y activos en clase. \\
\hline 2. Elusivo & $\begin{array}{l}\text { Muestran poco o nulo interés en la clase. Prefieren no participar } \\
\text { en clase y casi no preguntan. }\end{array}$ \\
\hline 3. Competitivo & $\begin{array}{l}\text { Su interés es demostrar ser el mejor en la clase. Gustan de los } \\
\text { reconocimientos por sus esfuerzos. }\end{array}$ \\
\hline 4. Colaborativo & $\begin{array}{l}\text { Gustan de trabajar con los demás aprendices. Aportan ideas para } \\
\text { el aprendizaje en común. }\end{array}$ \\
\hline 5. Dependiente & $\begin{array}{l}\text { Prefieren que les digan qué y cómo hacer las cosas. Presentan } \\
\text { poca curiosidad intelectual o escepticismo. }\end{array}$ \\
\hline
\end{tabular}

Fuente: Elaboración Propia.

En las preferencias del procesamiento de la información, Peterson y Kolb (2017) identifican nueve estilos de aprendizaje con base en la forma en que las personas perciben y procesar la información. Sin embargo, es importante señalar que el modelo anterior está basado en la teoría del aprendizaje experiencial de Kolb (1984). En el modelo original (ver Figura 1), el autor identifica cuatro estilos que conforman los cuadrantes de un plano cartesiano: en la parte superior del plano se ubican los estilos asociados con la experiencia concreta: acomodador y divergente; y en la parte inferior con la conceptualización abstracta, se ubican el convergente y el 
asimilador. En el extremo izquierdo se ubica la experimentación activa que es compartida por el acomodador y el convergente. En el extremo derecho que tiene que ver con la observación reflexiva se ubican los estilos divergente y asimilador.

Figura 1 - Estilos de aprendizaje de David Kolb

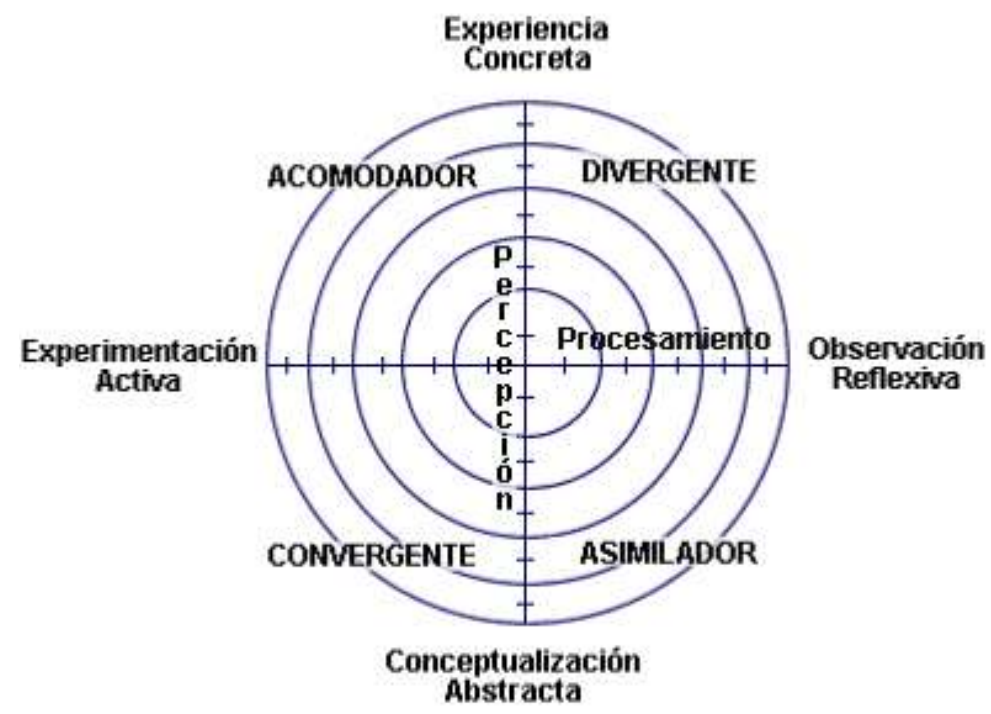

Fuente: Elaboración propia.

Cada estilo presenta ciertas características que tipifican al estudiante con respecto a sus preferencias para percibir y procesar información. Algunas de las más relevantes tienen que ver con la planeación y la acción. Por ejemplo, los divergentes y los asimiladores prefieren reflexionar antes de actuar; mientras que, los convergentes y los acomodadores gustan más por poner manos a la obra.

En las preferencias de la personalidad, se resaltan los trabajos del psicólogo Carl Jung (1971) reinterpretados por Briggs (1987). En este modelo se identifican dieciséis tipos de personalidad que determinan los estilos con base en la combinación de cuatro preferencias posibles con dos polos opuestos cada una (Tabla 2). 
Tabla 2 - Preferencias en la personalidad del modelo de Myers-Briggs (1987)

\begin{tabular}{|l|l|l|}
\hline DIMENSIONES & VALOR 1 & VALOR 2 \\
\hline $\begin{array}{l}\text { Dirección de la } \\
\text { energía }\end{array}$ & $\begin{array}{l}\text { Extroversión (E) - enfocada a la } \\
\text { gente y a las cosas }\end{array}$ & $\begin{array}{l}\text { Introversión (I) - enfocada a las } \\
\text { ideas y a la información }\end{array}$ \\
\hline Procesamiento & $\begin{array}{l}\text { Sensorial (S) - enfocado a los } \\
\text { hechos reales }\end{array}$ & $\begin{array}{l}\text { Intuición (I) - enfocado a las } \\
\text { posibilidades }\end{array}$ \\
\hline $\begin{array}{l}\text { Toma de } \\
\text { decisiones }\end{array}$ & $\begin{array}{l}\text { Pensamiento (Pe) enfocada a la } \\
\text { lógica }\end{array}$ & $\begin{array}{l}\text { Sentimiento (S) - enfocada a los } \\
\text { valores }\end{array}$ \\
\hline Estilo de vida & $\begin{array}{l}\text { Juicio (J) - enfocado a lo bien } \\
\text { estructurado }\end{array}$ & Percepción (P) - enfocado al ritmo \\
\hline
\end{tabular}

Fuente: Elaboración propia.

La combinación de uno de los valores con otro de las tres dimensiones siguientes, dan como resultado un estilo: por ejemplo, una persona puede ser extrovertida, intuitiva, con sentimiento y percepción, lo cual le daría un estilo de personalidad EISP; o introvertida, intuitiva, con sentimiento y percepción, lo cual resulta en IISP. La elección en un solo valor de una dimensión tipifica comportamientos diferentes en un estilo.

Por su parte, el Quirón Test fue el resultado de una propuesta teórica (Tabla 3) que retoma aspectos relevantes de varias de las teorías de estilos de aprendizaje encontradas en la literatura especializada, con la diferencia de enfocar aquellas preferencias que pueden aplicar por igual a ambientes a distancia y no solo presenciales (Lozano Rodríguez, Tijerina Salas \& García Cué, 2016). Entre ellas, se resaltan los trabajos de Witkin y Goodenough (1981) para resaltar las preferencias en la percepción con la teoría de la dependencia e independencia de campo.

Tabla 3 - Dimensiones consideradas en el Quirón Test

\begin{tabular}{|l|l|l|}
\hline Preferencias en la percepción & Global & Analítico \\
\hline Nivel de autonomía & Autónomo & Heterónomo \\
\hline Orientación & Teórico & Práctico \\
\hline Preferencias sensoriales & Visual & Verbal \\
\hline
\end{tabular}

La dimensión del nivel de autonomía fue considerada tomando en cuenta el modelo de dependencia e independencia propuesto por Grasha (2002). La orientación de teórico y práctico fue derivada del modelo de aprendizaje experiencial de Kolb (1984) en el eje del procesamiento que va de la 
experimentación activa (práctico) a la observación reflexiva (teórico). Por último, en las preferencias sensoriales se retoman algunos aportes de la teoría de los Dunn (1998) y de la teoría del VARK, enunciada por Fleming y Mills (1992) en lo referentes a la dualidad visual y verbal dejando de lado lo auditivo y lo kinestésico.

La inteligencia emocional fue originalmente descrita por Salovey y Mayer (1990) como la habilidad de reconocer las emociones y sus relaciones, y para participar en el razonamiento y la resolución de problemas sobre la base de las emociones. Posteriormente, el concepto fue popularizado por Goleman (1995) desde una perspectiva más pragmática. La idea de poder incidir en el propio comportamiento, a través de la toma de conciencia de las emociones, abría un campo fértil para su consideración en la vida cotidiana, laboral y profesional.

La inteligencia emocional tiene una gran influencia en los estudiantes, tanto en el contexto educativo como en el personal y el déficit en esta habilidad es causante de múltiples problemas académicos y psicosociales (Extremera-Pacheco \& Fernández-Berrocal, 2004). Goleman (1995) propuso un modelo de cinco dimensiones que incluía el autoconocimiento, la autorregulación, la autoeficacia, la empatía y las habilidades sociales. El autoconocimiento implica percibir las emociones propias, el impacto que tienen y emplearlas para tomar decisiones (GOLEMAN, MCKEE \& BOYATZIS, 2005). La autorregulación es un proceso voluntario que incluye la fijación de metas, el monitoreo y la planeación (ROESER \& PECK, 2009). La empatía es, además de ponerse en los zapatos de los demás, la habilidad para identificar los pensamientos y sentimientos de otros y responder a estos de manera apropiada (BARON-COHEN, 2011). La autoeficacia es una estimación propia de la capacidad para realizar una actividad con éxito (PALIS \& GREEN, 2001). Para Segrin y Taylor (2007) las habilidades sociales se definen como la capacidad de interactuar con los demás de una manera que sea apropiada y efectiva. Muchas personas se han preguntado por qué socializan más fácilmente con ciertas personas y más difícilmente con otras.

Mestre (2003) propuso un cuestionario para medir cuatro dimensiones de la Inteligencia Emocional, a saber: autoconocimiento, autorregulación, autoeficacia y empatía. Con base en modelos previamente expuestos sobre el concepto de 
inteligencia por autores como la inteligencia componencial de Sternberg (1982), inteligencias múltiples de Gardner (1983), inteligencia emocional Salovey y Mayer (1990) y la conceptualización pragmática de la inteligencia emocional de Goleman (1995).

Se partió del supuesto de que los estudiantes en ambientes escolares, pueden llegar a ser más efectivos en el aprovechamiento académico, cuando son capaces de lidiar con sus propias emociones y con las de otros (GOLEMAN, 1995). Aunado a lo anterior, surge la idea de si las preferencias para aprender están relacionadas de alguna manera con el manejo y control que tienen las personas sobre sus propias emociones.

A continuación, se identificaron algunas relaciones entre los estilos de aprendizaje y la inteligencia emocional.

Paloff y Pratt (2003) explicaron que el desempeño académico de los estudiantes que toman cursos en la modalidad a distancia, se puede ver afectado por las preferencias que manifiestan estos últimos en lo relacionado al uso de la tecnología y no solamente a sus preferencias instruccionales, psicológicas o. Lozano Rodríguez (2016) explicó que los estilos de aprendizaje han sido conceptualizados en ambientes presenciales y son pocos los trabajos de investigación que se han llevado a cabo para identificar estilos en ambientes de educación a distancia o ambientes en línea (LOZANO RODRIGUEZ, 2016).

Aunado a lo anterior, los constructos que conforman la inteligencia emocional han sido considerados como piezas clave para llevar a cabo un buen desarrollo socioemocional en las personas (GOLEMAN, 1995). Aunque existen autores que señalan la noción de inteligencia emocional de distintas maneras, todos coinciden de alguna manera en que se requiere de competencias emocionales para resolver problemas adaptativos del entorno (FRAGOSO-LUZURIAGA, 2015).

Alavinia y Ebrahimpour (2012) correlacionaron tanto los estilos de aprendizaje basados en la teoría del VAK (preferencias sensoriales) como la inteligencia emocional con el test de Bar-On (1997) en estudiantes iraníes que estaban aprendiendo inglés como segunda lengua. Sus resultados arrojaron que había una correlación significativa de 0.66 entre ambos constructos. Sin embargo, lo relevante fue que, en términos de sexo, las mujeres tenían una media superior a los 
hombres, lo cual puede indicar que las mujeres tienen un mejor desempeño en términos de su inteligencia emocional con respecto a los hombres. Por su parte, Afshar, Tofighi y Hamazavi (2016) también realizaron un estudio sobre las relaciones entre estilos de aprendizaje, estrategias de aprendizaje e inteligencia emocional con alumnos iraníes que estaban aprendiendo inglés como segundo idioma. Sin embargo, el modelo de estilos de aprendizaje utilizado fue el propuesto por Kolb (1984). Los resultados arrojaron que no hubo relación estadísticamente significativa entre los estilos de aprendizaje y la inteligencia emocional, pero sí entre las estrategias de aprendizaje y la inteligencia emocional. El aprovechamiento académico estuvo relacionado significativamente con la inteligencia emocional y con el uso de estrategias de aprendizaje y no así con los estilos de aprendizaje.

Leasa, Corebima, Ibrohim y Suwonoc (2017) llevaron a cabo un estudio que comparaba estudiantes con diferentes estilos de aprendizaje, basados en la teoría del VARK (FLEMING \& MILLS, 1992) sobre la afectación en su inteligencia emocional, basado en el constructo propuesto por Goleman (1995). Los resultados indicaron que los aprendices kinestésicos demostraban un mayor nivel de inteligencia emocional que los aprendices auditivos y lectores. En el campo de los estilos de aprendizaje basados en los hemisferios cerebrales, Estrada, Monferrer y Moliner (2019) realizaron un estudio con estudiantes universitarios con respecto al desarrollo de la inteligencia emocional. Sus resultados arrojaron que los estilos de aprendizaje asociados a las dominancias mixtas influyen de manera positiva en el desarrollo de la inteligencia emocional. Además, los estudiantes con estilos de aprendizaje asociados al hemisferio derecho tienen un mayor desarrollo de la inteligencia emocional.

Después de la revisión de la información anterior, se detectó que hay poca información sobre trabajos que relacionaran los Estilos de Aprendizaje en alumnos que trabajan en modalidades apoyadas de TIC -como la semipresencial y a distanciay diversas dimensiones de Inteligencia Emocional. Por eso, surgió la siguiente pregunta: ¿Qué relación existe entre los estilos de aprendizaje en ambientes a distancia y la inteligencia emocional en alumnos de pregrado, en especial en una Universidad del Sur de Sonora, México? 
Para contestar esta pregunta, se propuso una investigación con el objetivo de relacionar los Estilos de Aprendizaje en ambientes a distancia con algunos factores de Inteligencia Emocional de alumnos de pregrado de una Universidad del Sur de Sonora.

El supuesto planteado fue que las preferencias en cuanto a los Estilos de Aprendizaje en ambientes a distancia tienen una fuerte relación con cuatro dimensiones de Inteligencias Múltiples en alumnos de pregrado.

La investigación fue planteada para la identificación tanto de estilos de aprendizaje como de Inteligencia Emocional de alumnos que trabajan en modalidades a distancia utilizando las propuestas de Lozano Rodríguez, Tijerina Salas \& García Cué (2016) y Mestre (2003), la correlación existente entre los datos, así como factores que pueden influir (género y el tipo de estudios). Todo la anterior como punto de partida para el planteamiento de estrategias didácticas adecuadas para los alumnos de una Universidad del Sur de Sonora.

\section{Materiales y métodos}

El tipo de investigación fue de tipo cuantitativa, no probabilística, descriptiva y correlacional.

La Población se integró por alumnos matriculados en el semestre agostodiciembre 2018 de una Universidad pública del Sur de Sonora, México. La muestra fue seleccionada de manera no probabilística por 193 estudiantes de dieciséis carreras, siete de licenciatura y nueve de ingeniería.

Se utilizaron dos instrumentos para la recolección de información:

- Estilos de aprendizaje. Se utilizó el Quiron Test (LOZANO RODRÍGUEZ, TIJERINA SALAS \& GARCÍA CUÉ, 2016) que mide los estilos de aprendizaje mediante cuatro dimensiones bipolares: preferencia en la percepción (analítica, global), nivel de autonomía (heterónomo, autónomo), orientación (teórico y práctico) y Preferencias sensoriales (visual, verbal), (eje., Me gusta que las actividades tengan un orden lógico). Consta de 56 ítems en formato de respuesta tipo Likert con opciones desde 1 (totalmente en desacuerdo) hasta 6 (totalmente de acuerdo).

- Inteligencia emocional. Se empleó el del Cuestionario de Inteligencia Emocional (CIE) (MESTRE, 2003) que mide cuatro dimensiones de este 
constructo: autoconocimiento, involucra el conocimiento de estados emocionales, habilidades metacognitivas y la apertura mental, empatía, autorregulación y autoeficacia (eje., Cuando estoy muy enfadado no me paro a pensar sobre lo que hago o digo.). Consta de 56 ítems de respuesta tipo Likert con opciones desde 1 (totalmente en desacuerdo) hasta 6 (totalmente de acuerdo).

Además, se añadieron variables socioacadémicas como edad, genero, programa académico, semestre, si contaban o no con computadora y si tenían o no acceso a Internet.

Los instrumentos se aplicaron en diferentes momentos en un lapso de cinco días de dos campus diferentes de la misma universidad en el mes de septiembre de 2018. Se les pidió a los discentes que contestaran de manera voluntaria los cuestionarios después de una breve explicación.

Los datos se analizaron a través de estadísticos descriptivos univariados y pruebas de normalidad de Kolmogorov-Smirnov $(\alpha=0.05)$ por ser una muestra de 193 alumnos. Después, se seleccionaron pruebas no paramétricas de correlación de Spearman y de comparación de dos muestras independientes de U de MannWhitney- Wilcoxon $(\alpha=0.05)$ por género y por tipo de estudios (licenciatura / ingeniería). Los análisis se apoyaron del paquete estadísticos IBM SPSS V24.0 bajo el sistema operativo Windows.

\section{Resultados}

Se analizaron a 193 discentes. El 57.5\% de los alumnos fue de género femenino y el $42.5 \%$ del masculino. El intervalo de edades de los alumnos estuvo comprendido entre los 18 y 28 años. La edad promedio fue de 20.60, teniendo la mayoría de ellos entre 18 y 23 años. El coeficiente de variación fue del 9\% que indica que las edades de los encuestados fueron muy similares. Se detectaron 53.9\% de estudiantes de Licenciatura y 46.1\% de Ingeniería.

En esta investigación, contestaron los dos cuestionarios discentes de 16 carreras: siete de licenciatura (Psicología, Ciencias de la Educación, Administración, Educación infantil, Diseño Gráfico, Ejercicio Físico, así como Gestión y Desarrollo 
del arte) y nueve de ingeniería (Civil, Industrial, Software, Biosistemas, Biotecnología, Químico, Mecatrónica, Electromecánica y Electrónica). La figura 2 muestra el porcentaje de la participación de los participantes en esta investigación. Se destaca que el 16\% de los encuestados fueron de la licenciatura en Psicología, 16\% de Ciencias de la Educación, y el 11\% de Administración. En cuanto a los alumnos de Ingeniería se resaltan el 8.3\% de Industrial, 8.3\% de Biotecnología y 5.7\% de Química.

Figura 2 - Alumnas y alumnos encuestados de distintas carreras

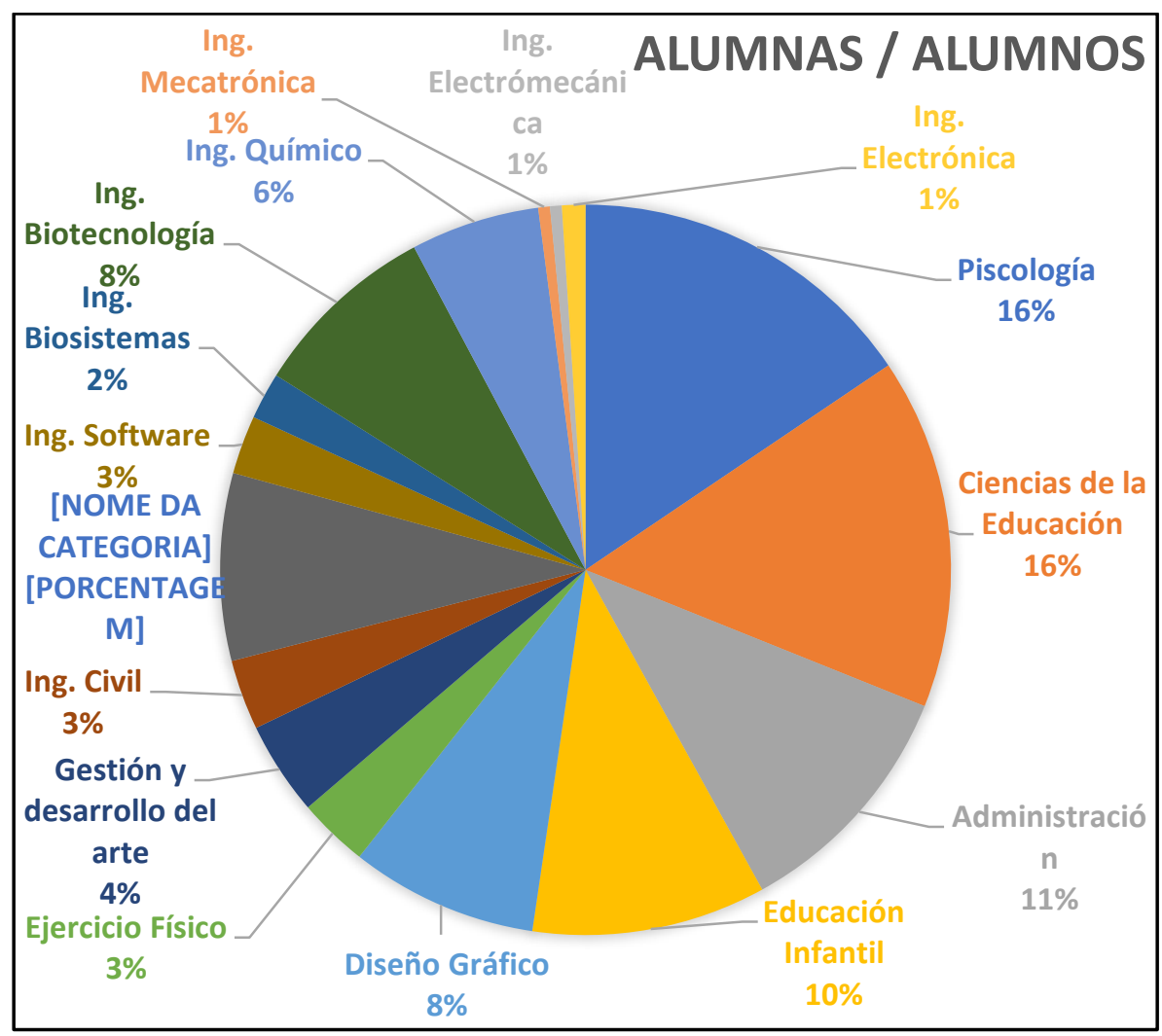

El 91\% de los entrevistados cuenta con computadora y el $92 \%$ con acceso a Internet. También, se realizó un análisis para la identificación del número de los discentes encuestados por género. Se pudo constatar que hay mayor número de alumnas de las carreras de licenciatura, y de alumnos de las carreras de ingeniería.

Los resultados de estadísticos descriptivos de los Estilos de Aprendizaje se muestran en la Tabla 4. 
Tabla 4 - Estadísticos descriptivos de los Estilos de Aprendizaje

\begin{tabular}{|l|l|l|l|l|l|l|l|l|}
\hline Dimensión & & Mín & Máx & Media & Mediana & $\begin{array}{l}\text { Desviación } \\
\text { Estándar }\end{array}$ & Varianza & $\begin{array}{l}\text { Coeficiente de } \\
\text { Variación }\end{array}$ \\
\hline $\begin{array}{l}\text { Preferencias } \\
\text { de } \\
\text { percepción }\end{array}$ & Analítico & 15 & 42 & 31.83 & 32 & 6.29 & 39.559 & 19.76 \\
\cline { 2 - 9 } & Global & 9 & 42 & 32.34 & 33 & 6.434 & 41.391 & 19.89 \\
\hline $\begin{array}{l}\text { Nivel de } \\
\text { autonomía }\end{array}$ & Dependiente & 15 & 42 & 32.31 & 33 & 6.343 & 40.236 & 19.63 \\
\cline { 2 - 9 } & Independiente & 16 & 42 & 32.04 & 32 & 5.697 & 32.457 & 17.78 \\
\hline \multirow{2}{*}{ Orientación } & Teórico & 21 & 42 & 33.37 & 34 & 5.89 & 34.692 & 17.65 \\
\cline { 2 - 9 } & Práctico & 19 & 42 & 33.34 & 34 & 5.996 & 35.955 & 17.98 \\
\hline \multirow{2}{*}{$\begin{array}{l}\text { Preferencias } \\
\text { sensoriales }\end{array}$} & Visual & 19 & 42 & 32.2 & 33 & 5.917 & 35.016 & 18.37 \\
\cline { 2 - 9 } & Verbal & 14 & 42 & 32.65 & 33 & 5.567 & 30.99 & 17.05 \\
\hline
\end{tabular}

En la tabla se puede observar que la mayoría de los valores promedio son altos en las cuatro dimensiones, el coeficiente de variación indica que las respuestas de alumnas y alumnos de la universidad es similar.

La figura 3 muestra el promedio de las preferencias de las ocho dimensiones de los Estilos de Aprendizaje. Los discentes tienen mayor preferencia teórica.

Figura 3 - Promedio de Estilos de Aprendizaje de alumnas y alumnos

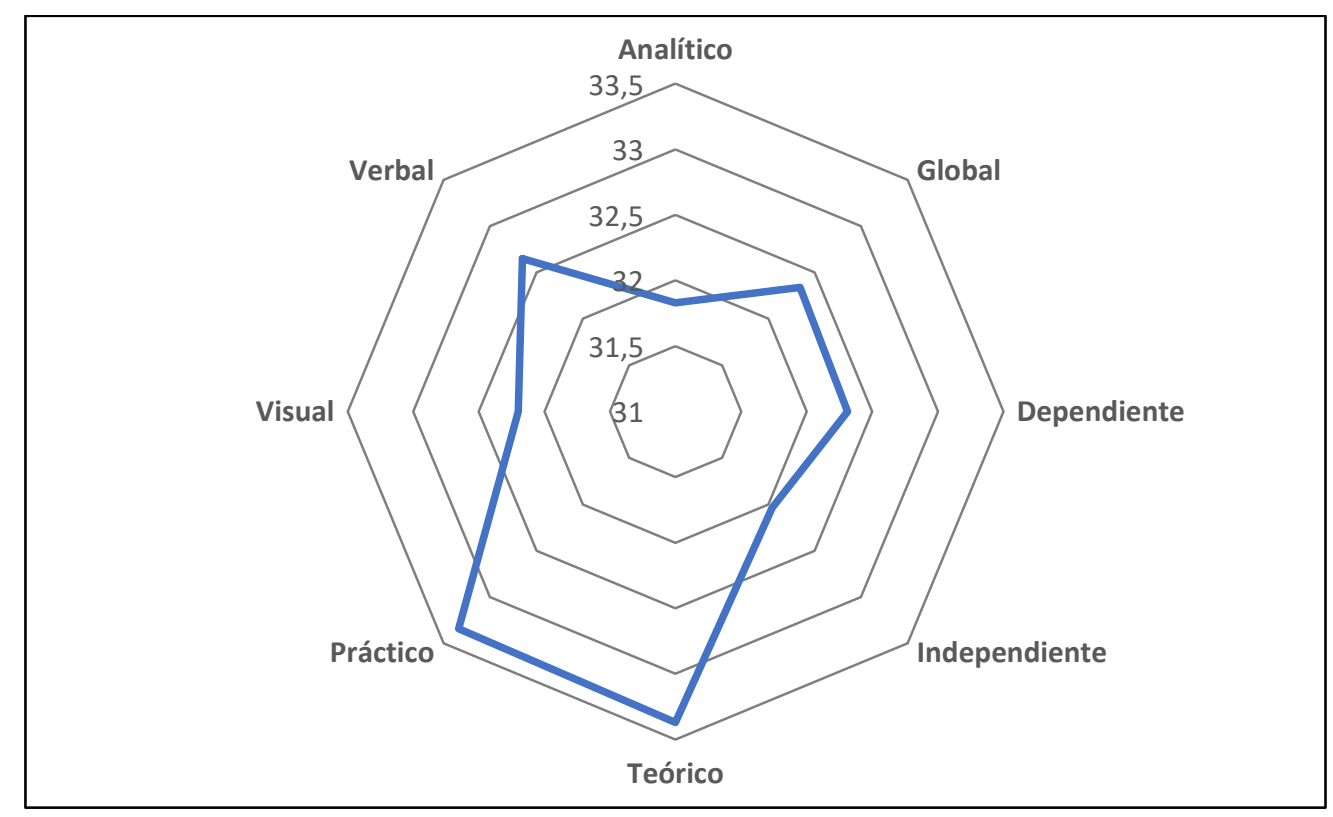

De acuerdo con Lozano et al. (2016, p. 266-267) y a los resultados obtenidos, las cuatro dimensiones expresan:

- Preferencias de percepción Globales: los alumnos y las alumnas son sensibles y usan su intuición. Se orientan mucho a la socialización con las personas y 
menos a las tareas. Además, procuran la esencia de lo que aprenden. Se recomienda que trabajen de manera colaborativa con otros alumnos, que hagan proyectos, resuelvan problemas y se les propongan retos.

- Nivel de Autonomía Dependientes: los discentes requieren de un profesor o compañero que los guíe. Además, son un poco inseguros ya que requieren que se les diga lo que está bien o mal. También, son personas cálidas, sensibles e influenciables ya que construyen sus ideas y sus posturas de otras personas. Se recomienda que trabajen en equipos donde puedan seguir a un líder y brindarle el apoyo en todo momento.

- Orientación Teóricos: los estudiantes prefieren los ensayos, las explicaciones y las teorías. Suelen utilizar un nivel de vocabulario elevado y presentan problemas para aterrizar las ideas. Se recomienda trabajar con el uso de casos de estudio, el aprendizaje basado en problemas y situaciones que reten el intelecto.

- Preferencias sensoriales Verbales: los alumnos y las alumnas disfrutan del uso de la palabra de manera presencial o por el uso de chats, foros de discusión o aplicaciones de celulares como el WhatsApp. También, les interesan archivos que contienen audio como un elemento primordial. Se recomienda que trabajen en debates, conferencias y el uso de archivos de audio.

Los resultados de estadísticos descriptivos de las puntuaciones obtenidas por los discentes en el Cuestionario de Inteligencia emocional se muestran en la Tabla 5.

Tabla 5 - Estadísticos descriptivos del Cuestionario de Inteligencia Emocional (CIE)

\begin{tabular}{|l|l|l|l|l|l|l|l|}
\hline Inteligencia & Mín & Máx & Media & Mediana & $\begin{array}{l}\text { Desviación } \\
\text { estándar }\end{array}$ & Varianza & $\begin{array}{l}\text { Coeficiente } \\
\text { de variación }\end{array}$ \\
\hline Autoconocimiento & 29 & 80 & 62.04 & 60 & 9.867 & 97.352 & $15.90 \%$ \\
\hline Autoregulación & 14 & 55 & 38.11 & 38 & 9.509 & 90.414 & $24.95 \%$ \\
\hline Autoeficacia & 21 & 49 & 38.47 & 38 & 5.856 & 34.292 & $15.22 \%$ \\
\hline Empatía & 20 & 45 & 33 & 31 & 6.731 & 45.313 & $20.39 \%$ \\
\hline
\end{tabular}


De acuerdo con los datos de la tabla 5, los alumnos evaluaron las cuatro dimensiones del CIE con valores altos expresados en la media. Los coeficientes de variación de Autoconocimiento, Autoeficacia y Empatía explican que sus respuestas de los discentes son muy similares. Las opiniones son más dispersas en Autorregulación. La tendencia de las respuestas de los discentes, se muestran en la Figura 4.

En concordancia con Mestre (2003, p. 309-311) y a los resultados obtenidos en los cuestionarios de Inteligencia Emocional, cada una de las cuatro dimensiones expresan:

- Autoconocimiento: A los discentes, les cuesta un poco reconocer sus propias emociones. Algunos son conscientes tanto de sus fortalezas como de sus debilidades. Otros no valoran adecuadamente sus propias capacidades.

- Autorregulación: Se detectó que hay alumnos que pueden manejar de sus emociones, en especial la ira, el rencor. la ansiedad o la angustia, pero se detecta que otros no. La mayoría asume las responsabilidades de sus propias decisiones y pueden afrontar cambios.

- Autoeficacia: La mayoría de los alumnos no están tan motivados para hacer sus cosas, les cuesta un poco tomar la iniciativa y en ocasiones son poco optimistas.

- Empatía: A una gran parte de los alumnos les cuesta comprender a los demás, ser altruistas. También, no aprovechan las aptitudes de trabajar en grupo aprovechando la diversidad de las personas. Además, les cuesta trabajo detectar las emociones de los demás. 
Figura 4 - Puntuación de los alumnos en las cuatro dimensiones de IE

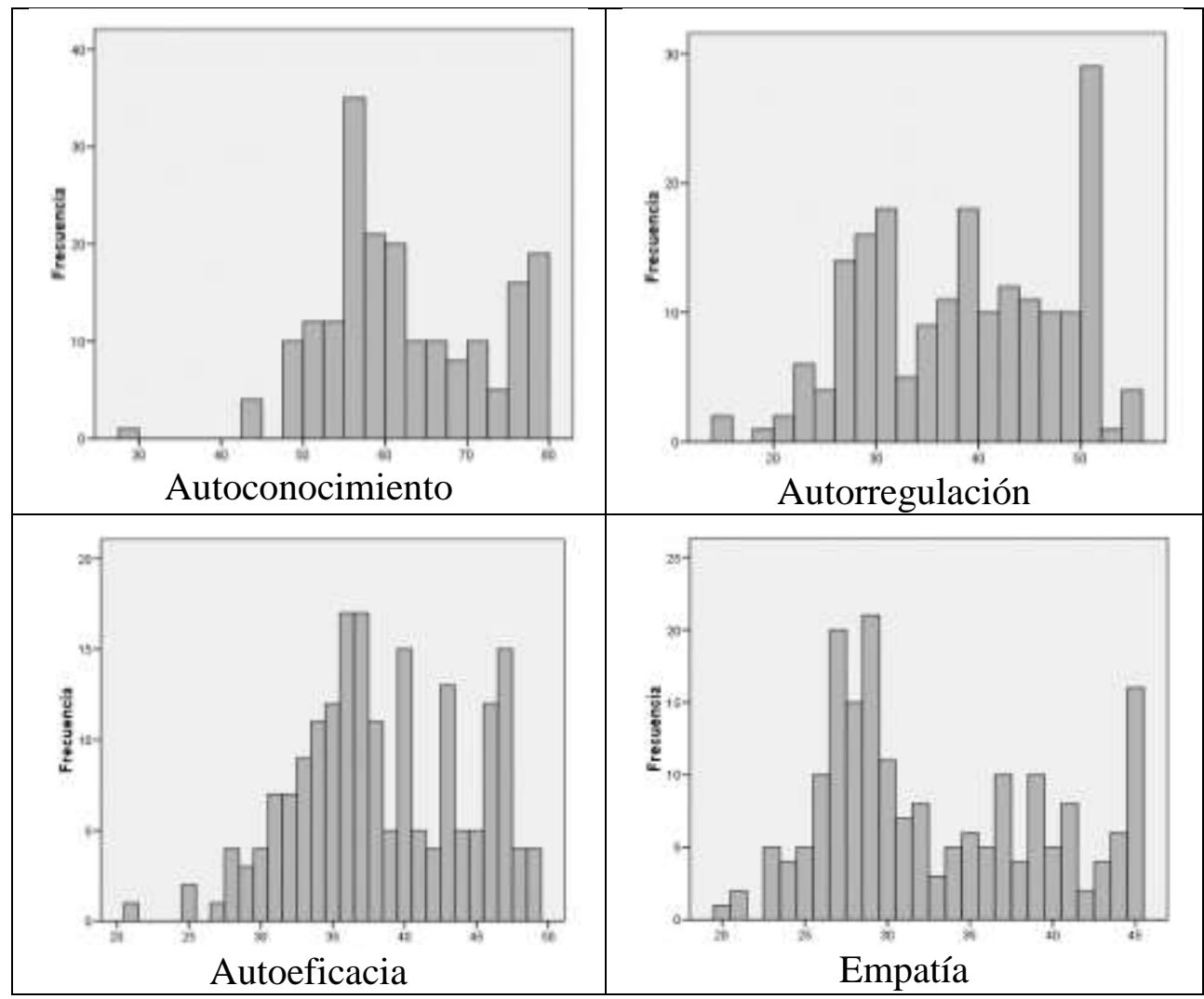

Mas adelante, se aplicaron pruebas de Normalidad a cada una de las variables. Como $n=193$ se utilizó la prueba de Kolmogorov-Smirnov $(\alpha=0.05)$. En un primer momento a las variables socio académicas, luego a las dimensiones de Estilos de aprendizaje y finalmente a las dimensiones de la Inteligencia Emocional. Muchas de las variables tienen un comportamiento no normal porque los Pvalue $\leq 0.05$, por ejemplo edad $(Z=0.146$ Pvalue $=0.000)$, semestre $(Z=0.238$ Pvalue $=0.000)$, si tienen computadora $(Z=0.534$ y Pvalue=0.000), analítico $(Z=0.087$ y Pvalue=0.001) y Autoconocimiento $(\mathrm{Z}=0.113$ y Pvalue=0.000).

Por lo anterior, se decidió emplear estadística no paramétrica en especial el análisis de correlación de Spearman $(\alpha=0.05)$ y los contrastes de dos muestras independientes de U de Mann-Whitney - Wilcoxon $(\alpha=0.05)$ tanto por género como por estudios de licenciatura o Ingeniería.

El análisis de correlación de Spearman se hizo con las ocho dimensiones de estilos de aprendizaje con las cuatro dimensiones de inteligencia emocional (Tabla 6). 
Tabla 6 - Correlaciones entre estilos de aprendizaje e inteligencia emocional

\begin{tabular}{|l|l|l|l|}
\hline Variables & Rho & Pvalue & Significancia \\
\hline Analítico - Autoconocimiento & -0.149 & 0.039 & $\star$ \\
\hline Analítico - Autorregulación & -0.271 & 0.000 & $\star \star$ \\
\hline Analítico - Autoeficacia & -0.169 & 0.019 & $\star$ \\
\hline Analítico - Empatía & -0.324 & 0.000 & $\star \star$ \\
\hline Global - Autoconocimiento & -0.201 & 0.005 & $\star$ \\
\hline Global - Autorregulación & -0.284 & 0.000 & $\star \star$ \\
\hline Global - Autoeficacia & -0.228 & 0.001 & $\star \star$ \\
\hline Global - Empatía & -0.404 & 0.000 & $\star \star$ \\
\hline Dependiente - Autoconocimiento & -0.211 & 0.003 & $\star \star$ \\
\hline Dependiente - Autorregulación & -0.289 & 0.000 & $\star \star$ \\
\hline Dependiente - Autoeficacia & -0.228 & 0.001 & $\star \star$ \\
\hline Dependiente - Empatía & -0.404 & 0.000 & $\star \star$ \\
\hline Independiente - Autorregulación & -0.280 & 0.000 & $\star \star$ \\
\hline Independiente - Autoeficacia & -0.152 & 0.035 & $\star$ \\
\hline Independiente - Empatía & -0.394 & 0.000 & $\star \star$ \\
\hline Teórico - Autoconocimiento & -0.210 & 0.003 & $\star \star$ \\
\hline Teórico - Autorregulación & -0.286 & 0.000 & $\star \star$ \\
\hline Teórico - Autoeficacia & -0.220 & 0.002 & $\star \star$ \\
\hline Teórico - Empatía & -0.378 & 0.000 & $\star \star$ \\
\hline Práctico - Autoconocimiento & -0.178 & 0.013 & $\star$ \\
\hline Práctico - Autorregulación & -0.283 & 0.000 & $\star \star$ \\
\hline Práctico - Autoeficacia & -0.222 & 0.002 & $\star \star$ \\
\hline Práctico - Empatía & -0.354 & 0.000 & $\star \star$ \\
\hline Visual - Autorregulación & -0.207 & 0.004 & $\star \star$ \\
\hline Visual - Autoeficacia & -0.146 & 0.042 & $\star$ \\
\hline Visual - Empatía & -0.334 & 0.000 & $\star \star$ \\
\hline Verbal - Autorregulación & -0.177 & 0.014 & $\star$ \\
\hline Verbal - Empatía & -0.259 & 0.000 & $\star \star$ \\
\hline
\end{tabular}

Los estudiantes que obtuvieron mayores valores en los Estilos de Aprendizaje Analítico, Global, Dependiente, Teórico y Práctico obtienen menores valores en las cuatro dimensiones de Inteligencia Emocional. Aquellos que obtuvieron mayores puntuaciones en el Estilo de Aprendizaje Verbal tienen menores valores en Autorregulación y Empatía. Mientras que los que obtuvieron mayores puntuaciones en el Estilo de Aprendizaje Visual obtienen menores puntuaciones en las Inteligencias de Autorregulación, Autoeficacia y Empatía.

En las pruebas $U$ de Mann-Whitney-Wilcoxon para dos muestras independientes con $(\alpha=0.05)$ por género en los Estilos de Aprendizaje no se detectaron diferencias significativas, esto es, tanto alumnas como alumnos tienen dimensiones de estilos de aprendizaje similares. 
Los resultados de las pruebas U de Mann-Whitney- Wilcoxon para dos muestras independientes con $(\alpha=0.05)$ por género en Inteligencia Emocional se muestran en la tabla 7.

Tabla 7 - Pruebas de U de Mann-Whitney por género en Inteligencia Emocional

\begin{tabular}{|l|l|l|l|l|}
\hline & Autoconocimiento & Autorregulación & Autoeficacia & Empatía \\
\hline U de Mann-Whitney & 3694.000 & 3259.000 & 3698.000 & 3301.500 \\
\hline W de Wilcoxon & 7097.000 & 6662.000 & 7101.000 & 6704.500 \\
\hline Z & -2.236 & -3.371 & -2.228 & -3.264 \\
\hline Sig. asintót. (bilateral) & .025 & .001 & .026 & .001 \\
\hline
\end{tabular}

De acuerdo con lo obtenido en la tabla 7 se distinguen diferencias altamente significativas por género en Autorregulación y Empatía, esto es, las alumnas tienen más altas puntuaciones que los alumnos. En Autoconocimiento y Autoeficacia se distinguieron diferencias significativas y también las alumnas obtienen mayores puntuaciones que los alumnos.

La tabla 8 muestra los resultados de las pruebas U de Mann-WhitneyWilcoxon para dos muestras independientes con $(\alpha=0.05)$ que resultaron significativos o altamente significativos por tipo de estudios (licenciatura / Ingeniería) en las dimensiones de Estilos de Aprendizaje.

Tabla 8 - Pruebas de U de Mann-Whitney por tipo de estudios en IE

\begin{tabular}{|l|l|l|l|l|l|l|}
\hline & Analítico & Global & Independiente & Teórico & Práctico & Visual \\
\hline U de Mann-Whitney & 3524.500 & 3539.000 & 3488.500 & 3246.500 & 3465.500 & 3684.000 \\
\hline W de Wilcoxon & 8984.500 & 8999.000 & 8948.500 & 8706.500 & 8925.500 & 9144.000 \\
\hline Z & -2.857 & -2.819 & -2.951 & -3.578 & -3.010 & -2.444 \\
\hline $\begin{array}{l}\text { Sig. Asintótotica } \\
\text { (bilateral) }\end{array}$ & .004 & .005 & .003 & .000 & .003 & .015 \\
\hline
\end{tabular}

Se detectó que los valores en seis de las ocho dimensiones de Estilos de Aprendizaje son mayores en estudios de ingeniería que en los de licenciatura.

La tabla 9 muestra los resultados de las pruebas U de Mann-WhitneyWilcoxon para dos muestras independientes con $(\alpha=0.05)$ que resultaron altamente significativos por tipo de estudios (licenciatura / Ingeniería) en las dimensiones de Inteligencia Emocional. 
Tabla 9 - Pruebas de U de Mann-Whitney por tipo de estudios en IE

\begin{tabular}{|l|l|l|l|l|}
\hline & Autoconocimiento & Autorregulación & Autoeficacia & Empatía \\
\hline U de Mann-Whitney & 2259.000 & 2023.500 & 2181.500 & 2099.500 \\
\hline W de Wilcoxon & 6264.000 & 6028.500 & 6186.500 & 6104.500 \\
\hline Z & -6.130 & -6.740 & -6.336 & -6.551 \\
\hline Sig. Asintótica (bilateral) & .000 & .000 & .000 & .000 \\
\hline
\end{tabular}

De la información obtenida y de la tabla 9 se puede constatar que hay mayores puntuaciones en las cuatro dimensiones en los discentes que estudian cualquier licenciatura que los de cualquier tipo de ingeniería.

\section{Discusión}

El propósito del presente estudio fue el mostrar si existe algún tipo de relación entre los ocho Estilos de Aprendizaje y las cuatro dimensiones de Inteligencia Emocional. Era de esperarse que ciertos estilos de aprendizaje pudieran puntuar de manera directamente proporcional con algunas de las dimensiones (si es que no con todas) de la Inteligencia Emocional. Algunos autores como Witkin y Goodenough (1981) ya señalaban ciertas características de personalidad asociadas con los estilos cognitivos basados en la dimensión de la percepción. Los dependientes de campo, aquellos que eran influenciados por su campo visual, solían manifestar mayor Empatía que los independientes de campo. Peterson y Kolb (2017) por su parte, reportan que los estilos asociados con la dimensión concreta del procesamiento de la información están más orientados hacia las personas. Aunque no mencionan directamente a la Empatía como tal, aluden a una mejor relación con los demás, en contraste con aquellos cuya dimensión es la abstracta en el procesamiento de la información. Lo anterior contrasta con los hallazgos del presente estudio en donde los estilos Global, Dependiente y Práctico obtuvieron menores valores en las cuatro dimensiones de la Inteligencia Emocional, incluyendo la Empatía. No obstante, los estilos Analítico y Teórico sí fueron coherentes con lo reportado con estos autores.

Por otra parte, llama mucho la atención que aquellos alumnos que obtuvieron mayores puntuaciones en el Estilo de Aprendizaje Verbal hayan 
obtenidos menores valores en Autorregulación y Empatía. Lo anterior contrasta con lo reportado por Herrera Fernández, Camacho Gutiérrez y Heredia Escorza (2016), quienes relacionaron la autorregulación con los estilos de aprendizaje propuestos por Alonso, Gallego y Honey (1994): reflexivo, analítico, activo y pragmático. Los estudiantes con estilos de aprendizaje Analíticos y Reflexivos correlacionaban más alto con la autorregulación que los otros dos estilos. Lo anterior puede sugerir que efectivamente los procesos de reflexión y análisis que suponen estos estilos van de la mano con la dimensión de la autorregulación. Sin embargo, es menester mencionar que los constructos de autorregulación que se mencionan en ambos estudios pudieran ser un tanto diferentes en sus conceptualizaciones teóricas respectivas.

Por otra parte, los estudiantes que obtuvieron mayores puntuaciones en el Estilo de Aprendizaje Visual obtuvieron menores puntuaciones en las Inteligencias de Autorregulación, Autoeficacia y Empatía. En contraste, Alavinia y Ebrahimpour (2012) encontraron que el Estilo de Aprendizaje Visual del modelo VARK sí correlacionaba de manera positiva con todas las dimensiones de Inteligencia Emocional en estudiantes iraníes. Si bien es cierto que los constructos son un tanto diferentes, en teoría, tanto el Quiron Test como el VARK consideran la misma fundamentación teórica para el estilo visual. Leasa, Corebima, Ibrohim y Suwonoc (2017) encontraron que los aprendices kinestésicos demostraban un mayor nivel de inteligencia emocional que los aprendices auditivos y lectores, pero descartaron a los visuales porque no hubo suficientes estudiantes que ostentaran ese estilo. Sus sugerencias van en la línea de averiguar qué es lo que pasa con la inteligencia emocional de los estudiantes en tanto sus estilos de aprendizaje van cambiando a lo largo del tiempo tal y como lo menciona Sternberg (1997).

Por último, los hallazgos sugieren una diferencia marcada en lo relacionado por género. Las estudiantes mujeres presentan fuertes diferencias con respectos a los hombres en prácticamente todas las dimensiones de la Inteligencia Emocional, pero no así en los Estilos de Aprendizaje. Lo anterior coincide con lo encontrado por Mestre (2003), quien menciona que efectivamente hay una diferencia por sexo en la inteligencia emocional, pero no en los estilos de aprendizaje. Las estudiantes mujeres puntuaron más alto en la Empatía y Autorregulación. Los hallazgos 
sugieren que las habilidades para ejercer procesos de regulación y monitoreo de emociones en los otros son mayores en las mujeres que en los hombres.

\section{Conclusiones}

De acuerdo con los datos obtenidos y el análisis realizado se puede concluir:

1) El objetivo de esta investigación se cumplió y el supuesto no se rechaza.

2) Hace falta indagar a mayor profundidad la relación existente entre los Estilos de Aprendizaje y las dimensiones de la Inteligencia Emocional. Si bien es cierto que los datos obtenidos sugieren una cierta relación, tal vez se requiere una investigación más de corte cualitativa que venga a complementar lo presentado en este estudio.

3) No se puede hablar de manera concluyente sobre las relaciones encontradas, en términos categóricos. No obstante, el presente estudio ha arrojado un poco de luz sobre cómo ciertos Estilos de Aprendizaje están más relacionados con ciertas dimensiones de la Inteligencia Emocional. La Empatía y la Autorrealización fueron las dimensiones que parecen tener más relación con los Estilos de Aprendizaje.

4) Las diferencias encontradas por género y por tipo de estudio abren nuevas rutas de investigación más en lo relacionado con la Inteligencia Emocional que con los Estilos de Aprendizaje. Lo anterior permite visualizar posibles estudios a futuro que permitan conocer un poco más sobre cómo las estudiantes mujeres obtienen este mayor desarrollo sobre sus compañeros varones. Además, ver los perfiles de alumnos que entran a ciertas carreras universitarias para proponer programas de estudio adecuados a las características de sus discentes.

\section{Referencias}

ALAVINIA, P. \& EBRAHIMPOUR, S. On the Correlation between Emotional Intelligence and Learning Styles: The Case of Iranian Academic EFL Learners. Theory and Practice in Language Studies, v. 2, n. 6, p. 1291-1299, 2012.

ALONSO, C., GALleGO, D. \& HONEY, P. Los estilos de aprendizaje. Procedimientos de diagnóstico y mejora. Bilbao: Mensajero, 1994. 
AFSHAR, H. S., TOFIGHI, S. \& HAMAZAVI, R. Iranian EFL learners' emotional intelligence, learning styles, strategy use, and their L2 achievement. Issues in Educational Research, v. 26, n. 4, p. 635-652, 2016.

ASSUNÇÃO, R. S. \& MATOS, P. M. Perspetivas dos adolescentes sobre o uso do Facebook: um estudo qualitativo. Psicologia em Estudo, v. 19, n. 3, p. 539-547. Doi https://dx.doi.org/10.1590/1413-73722133716. 2014.

BAR-ON, R. Bar-On emotional quotient inventory: User's manual. Toronto: Multi-Health Systems, 1997.

BARON-COHEN, S. Zero Degrees of Empathy. London: Allen Lane. 2011.

BETTIS-OUTLAND, H. y GUILLORY, M.D. Emotional intelligence and organizational learning at trade shows. Journal of Business \& Industrial Marketing, n. 1, p. 126. Doi https://doi-org.itson.idm.oclc.org/10.1108/JBIM-03-2017-0066. 2018.

BOYKIN, A. W., \& BAILEY, C. T. The role of cultural factors in school relevant cognitive functioning [microform]: synthesis of findings on cultural contexts, cultural orientations, individual differences. En: A. W. BOYKIN, C. T. BAILEY. [Baltimore, MD]: [Washington, DC]: Center for Research on the Education of Students Placed at Risk, Johns Hopkins University y Howard University, 2000.

BOTHAinA A. A., HAMOUDA, A. M.; ABDELlA, G. M. Modeling of student academic achievement in engineering education using cognitive and non-cognitive factors. Journal of Applied Research in Higher Education, n. 2, p. 178-198, 2019.

BRIGGS, K. C. Myers-Briggs type indicator. Form G. Palo Alto, CA.: Consulting Psychologists Press, 1987.

CASSIDY, S. Learning styles: An overview of theories, models, and measures. Educational Psychology, v. 24, n. 4, p. 419-444, 2004.

CORNEJO CHÁVEZ, R., \& REDONDO ROJO, J. M. Variables y factores asociados al aprendizaje escolar: una discusión desde la investigación actual. Estudios Pedagógicos, Valdivia, n. 2, p. 155, 2007. Doi https://doi-org.itson.idm.oclc.org/10.4067/S071807052007000200009 .

CURRY, L. An Organization of Learning Styles Theory and Construct. In: $67^{\text {th }}$ Annual Meeting of the American Educational Research Association, Montreal, April 11-15, 1983. Disponible en: $<$ http://eric.ed.gov/ERICWebPortal/search/detailmini.jsp?_nfpb=true\&_\&ERICExtSear ch_SearchValue_0=ED235185\&ERICExtSearch_SearchType_0=no\&accno=ED23 $5185>$.

DE LA HOZ, L., ACEVEDO, D., y TORRES, J. Uso de Redes Sociales en el Proceso de Enseñanza y Aprendizaje por los Estudiantes y Profesores de la Universidad Antonio Nariño, Sede Cartagena. Formación Universitaria, v. 8, n. 4, p. 77-84, 2015.

DUNN, R. y DUNN, K. The complete guide to the learning styles inservice system. Boston: Allyn \& Bacon, 1998. 
EMI KAKUSHI, L. y MARTÍNEZ ÉVORA, Y.D. Las redes sociales en la educación en enfermería: revisión integradora de la literatura. Revista Latino Americana de Enfermagem, $\mathrm{n}$. 24, p. 1-12, 2016.

ESTRADA, M., MONFERRER, D. \& MOLINER, M.A. The Relation between Learning Styles according to the Whole Brain Model and Emotional Intelligence: A Study of University Students. Estudios sobre Educación, n. 36, p. 85-111, 2019.

EXTREMERA PACHECO, N.; FERNÁNDEZ-BERROCAL, P. El papel de la inteligencia emocional en el alumnado: evidencias empíricas. REDIE. Revista Electrónica de Investigación Educativa, v. 6, n. 2, p. 1-17, 2004.

FLEMING, N. D. \& MILLS, C. Not Another Inventory, Rather a Catalyst for Reflection. To Improve the Academy, n. 11, p. 137-155, 1992.

FRAGOSO-LUZURIAGA, R. Inteligencia emocional y competencias emocionales en educación superior, ¿un mismo concepto? Revista Iberoamericana de Educación Superior, v. VI, n. 16, p. 110-125, 2015.

GARDNER, H. Frames of mind. The theory of multiple intelligences. New York: Basic Books, 1983.

GOLEMAN, D. Emotional intelligence: Why it can matter more than IQ? London: Bloomsbury, 1995.

GOLEMAN, D., MCKEE, A.; BOYATZIS, R. E. Primal leadership: Realizing the power of emotional intelligence. Boston: Harvard Business School Press, 2005.

GRASHA, A. Teaching with style. San Bernardino: Alliance, 2002.

GRIGORENKO, E. L.; STERNBERG, R. J. Styles of Thinking, Abilities, and Academic Performance. Exceptional Children, v. 63, n. 3, p. 295-312, 1997.

Doi https://doi.org/10.1177/001440299706300301.

GUILD, P. B.; GARGER, S. Marching to Different drummers. Alexandria, VA: ASCD, 1998.

HANIN, V.; VAN NIEUWENHOVEN, C. The influence of motivational and emotional factors in mathematical learning in secondary education. European Review of Applied Psychology, v. 66, n. 3, p. 127-138, 2016.

HERRERA-FERNÁNDEZ，K. A.; CAMACHO-GUTIÉRREZ，D. F.; HEREDIAESCORZA, Y. La relación entre estilos de aprendizaje, autodirección y el desempeño académico en estudiantes de música de una universidad mexicana. Revista Electrónica Complutense De Investigación En Educación Musical - RECIEM, n. 13, p. 1-21. Doi https://doi.org/10.5209/RECIEM.51493. 2016.

IBABE, I. Academic failure and child-to-parent violence: Family protective factors. Frontiers in Psychology, n. 7, p. 1-11, 2016.

JUNG, C.G. Psychological Types. London: Routledge. 1921/1971. 
KOLB, D. A. Experiential Learning: Experience as the Source of Learning and Development. Englewood Cliffs: Prentice-Hall, 1984.

LEASA, M.; COREBIMA, A. D.; IBROHIM, SUWONOC, H. Emotional intelligence among auditory, reading, and kinesthetic learning styles of elementary school students in Ambon-Indonesia. International Electronic Journal of Elementary Education, v. 10, n. 1, p. 83-91. 2017.

LOZANO RODRÍGUEZ, A., TIJERINA SALAS, A. y GARCÍA CUÉ, J. L. Implementación del instrumento Quirontest para medir estilos de aprendizaje en estudiantes de pregrado en línea. Revista de Estilos de Aprendiraje, v. 9, n. 17, p. $240-267,2016$.

MAYRING, P. Emotional and motivational aspects of learning-A discussion of new mixed methods studies. International Journal of Educational Research, 2007. doi https://doiorg.itson.idm.oclc.org/10.1016/j.ijer.2007.11.014. 2007.

MESTRE, J. Validación empírica de una prueba para medir la inteligencia emocional en una muestra de estudiantes de bahía de Cádiz. Tesis (Doctoral) - Universidad de Cádiz, España, Facultad de Ciencias de la Educación, Departamento de Psicología, 2003.

MOESTUE, H.; HUTTLY, S. Adult education and child nutrition: the role of family and community. Journal of Epidemiology and Community Health, v. 62, n. 2, p. 153-159, 2008.

PALIS, L. L.; GREEN, S. G. Leadership self-efficacy and managers motivation for leading change. Journal of Organizational Behavior, n. 23, p. 215-235. Doi http://dx.doi.org/10.1002/job.137. 2001.

PALOFF, R.M. y PRATT, K. Virtual student. A profile and guide to working with Online learners. San Francisco: Jossey-Bass, 2003.

PÉREZ ALCALÁ, M. S.; ORTIZ ORTIZ, M. G.; FLORES BRISEÑO, M. M. Redes sociales en Educación y propuestas metodológicas para su estudio. Ciencia, Docencia y Tecnología, v. 26, n. 50, p. 188-206, 2015.

PETERSON, K. y KOLB, D. How You Learn Is How You Live: Using Nine Ways of Learning to Transform Your Life. Oakland: Berrett-Koehler Publishers, 2017.

RAMÍREZ DORANTES, M. del C. Modelo causal de los factores asociados al aprendizaje autorregulado como mediador del rendimiento académico en estudiantes universitarios. 2016. Disponible en: <http://search.ebscohost.com.itson.idm.oclc.org/login.aspx?direct $=\mathrm{t}$ rue\&db=edsoai\&AN=edsoai.on1050992869\&lang=es\&site=eds-live $>$.

ROESER, R. W.; PECK, S. C. An Education in Awareness: Self, Motivation, and SelfRegulated Learning in Contemplative Perspective, Educational Psychologist, v. 44, n. 2, p. 119136, 2009. Doi: 10.1080/00461520902832376

SALOVEY, P. y MAYER, J. D. Emotional intelligence. Imagination, Cognition, and Personality, v. 9, n. 2, p. 185-211, 1990.

SEGRIN, C.; TAYLOR, M. Positive interpersonal relationships mediate the association between social skills and psychological well-being. Personality and Individual Differences, v. 43, n. 4, p. 637-646. doi 10.1016/j.paid.2007.01.017. 2007. 
STERNBERG, R. J. A Componential Approach to Intellectual Development. En: R. J. Sternberg (Ed.). Advances in the Psychology of Human Intelligence (p. 23-34). Hillsdale: Erlbaum, 1982.

STERNBERG, R. J. Thinking styles. New York: Cambrigde University Press, 1997.

TORRES VALENZUELA, A.; VELÁZQUEZ HERNÁNDEZ, G. Y.; MARTÍNEZ LUNA, A. A.; et al. Rasgos de personalidad en alumnos de reciente ingreso a la carrera de medicina. Investigación En Educación Médica, v. 25, p. 27-35, 2018.

ZUO, X.; WEAVER, R.; MACRAE, J.; WANG, L. How Much Do Emotional, Behavioral, and Cognitive Factors Actually Impact College Student Attitudes towards English Language Learning? A Quantitative and Qualitative Study. Information, v. 10, n. 5, p. 166, 2019. Doi: https://doi.org/10.3390/info10050166

WITKIN, H.; GOODENOUGH, D. Estilos cognitivos. Naturalezas y Orígenes. Madrid: Pirámide, 1981.

RECIBIDO: $22 / 01 / 2020$

APROBADO: $20 / 02 / 2020$

RECEIVED: 01/22/2020

APPROVED: 02/20/2020

RECEBIDO: $22 / 01 / 2020$

APROVADO: 02/20/2020 